\title{
Graft Patency in Long-term Survivors after Renovisceral Debranching with VORTEC
}

\author{
Anna Winklehner • Thi Dan Linh Nguyen-Kim • \\ Thomas Pfammatter - Zoran Rancic • \\ Dieter Mayer • Mario Lachat · Thomas Frauenfelder
}

Received: 28 March 2014/ Accepted: 30 June 2014/Published online: 6 September 2014

(C) Springer Science+Business Media New York and the Cardiovascular and Interventional Radiological Society of Europe (CIRSE) 2014

\begin{abstract}
Purpose This study was designed to evaluate retrospectively the long-term stent-graft patency after renovisceral revascularization with Viabahn Open Revascularization Technique (VORTEC) using computed tomography angiography (CTA) and magnetic resonance angiography (MRA).

Methods In 34 patients (seven women; mean age $72 \pm 8$ years) with aortic aneurysm, 63 renovisceral vessels (i.e., 54 renal, nine visceral arteries) were revascularized with VORTEC between 2004 and 2009. All patients obtained a pre- and postinterventional CTA and at least one follow-up CTA or MRA after 6 or more months following intervention (median follow up: 43 months). Detection of bypass occlusion, bypass stenosis, infolding, stent-graft
\end{abstract}

\author{
A. Winklehner - T. D. L. Nguyen-Kim - T. Pfammatter · \\ T. Frauenfelder $(\bowtie)$ \\ Institute of Diagnostic and Interventional Radiology, University \\ Hospital Zurich, Raemistrasse 100, 8091 Zurich, Switzerland \\ e-mail: Thomas.Frauenfelder@usz.ch \\ A. Winklehner \\ e-mail: anna.winklehner@usz.ch \\ T. D. L. Nguyen-Kim \\ e-mail: thidanlinh.nguyen@usz.ch \\ T. Pfammatter \\ e-mail: thomas.pfammatter@usz.ch \\ Z. Rancic - D. Mayer - M. Lachat \\ Clinic for Cardiovascular Surgery, University Hospital Zurich, \\ Raemistrasse 100, 8006 Zurich, Switzerland \\ e-mail: zoran.rancic@usz.ch \\ D. Mayer \\ e-mail: dieter.mayer@usz.ch \\ M. Lachat \\ e-mail: mario.lachat@usz.ch
}

fractures and dislocations, and kidney shrinkage were noted by two readers in consensus. Furthermore, mortality during follow-up was assessed using the medical report. Results During follow-up, $12.6 \%$ of stent-grafts occluded. Cumulative patency rate was $95.2 \pm 2.7 \%$ at 12 months, $87.7 \pm 4.4 \%$ at 24 and 36 months, and $84.7 \pm 5.2 \%$ at $48,60,72,84$, and 89 months, respectively. Overall, $19 \%$ of stent-grafts $(12 / 63)$ developed bypass stenosis $(<50 \%, 10$ stent-grafts; $>50 \%, 2$ stentgrafts), in one case stenosis ( $>70 \%$ ) was suspected to be hemodynamically significant. No secondary dislocation, no infolding of renovisceral stent-grafts, and no stent-graft fracture occurred. Kidney shrinkage occurred in nine patients, primarily in patients with an occluded Viabahn $(n=7)$. Eleven patients $(32.3 \%)$ died within the followup time period.

Conclusions In long-term survivors after VORTEC cumulative patency rate remained high, and no stent-graft fractures or secondary dislocations occurred.

Keywords Computed tomography angiography · VORTEC $\cdot$ Aortic aneurysm

\section{Introduction}

Treatment of thoracoabdominal aortic aneurysms (TAAA) and pararenal aortic aneurysms (PAAA) is complex. Open repair is considered the "gold standard" surgical procedure $[1,2]$; however, it involves wide aortic exposure, aortic cross-clamping, and thus leads to intraoperative ischemia of abdominal organs and spinal cord and poses a great risk even for "so-called" fit for surgery patients $[3,4]$. A hybrid procedure, performed through an abdominal approach, is considered less invasive than open repair and consists of 
surgical bypass to renovisceral arteries, ensuring perfusion while preserving adequate aortic landing zones for subsequent endovascular aneurysm repair [5]. Since the hybrid operation was first introduced in 1999 [6], different studies have shown encouraging results with acceptable mortality rates [7-10]; however, the procedure is time consuming and standard anastomoses to the renovisceral branches are technically demanding and prone to bypass related complications $[2,7,11]$. To simplify renovisceral debranching, a technique using self-expanding stent-grafts, Viabahn Open Revascularization Technique (VORTEC), has been developed [12, 13]. The technique is characterised by minimal dissection and a minimisation of blood flow interruption and thus reduction of ischemia [13]. The shortand mid-term results have shown a high patency rate of stent-grafts [7, 14]; however, long-term patency rates have not been documented. Thus, the purpose of this study was to retrospectively assess stent-grafts on follow-up computed tomography angiography (CTA) or magnetic resonance angiography (MRA) from 6 to 89 months (median 43 months) after renovisceral revascularization with VORTEC.

\section{Materials and Methods}

Patient Population and Study Design

Between August 2004 and March 2009, 73 patients with TAAA or PAAA underwent renovisceral revascularisation with VORTEC as the first step in part of the hybrid-procedure (i.e., 57 patients) or simultaneously with open graft repair (i.e., 16 patients), when the two-step procedure was not feasible (ruptured aneurysm, $n=6$; aneurysm morphologically unsuitable for endovascular repair, $n=10$ ). Patients with occlusive disease were not included in this study. At our institution, the protocol for follow-up includes CTA and clinical follow-up postinterventionally, at 6 weeks, at 3, 6, and 12 months, and then annually. Only patients with available pre- and postinterventional (within 1 month) CTA and at least one available follow-up CTA or MRA after 6 months or more following the intervention were included in this study. Twenty-five patients were lost to follow-up within 6 months and 14 patients died; 30-day mortality was $9.6 \%$ (7/73).

Thus, the remaining 34 patients (27 men and 7 women, mean age $72 \pm 8$ years) made up the study population. Five patients were symptomatic, and one had a ruptured aneurysm. All patients had at least one renovisceral artery revascularized with the VORTEC. This technique was used in 29 patients as part of the hybrid procedure to allow endovascular aneurysm repair in a second step (mean interval between both procedures: 24 days). In five patients with
Table 1 Patient characteristics

\begin{tabular}{ll}
\hline Total number of patients & 34 \\
Disease & 14 \\
TAAA & 3 \\
Type according to the modified Crawford classification [27] \\
Crawford I & 2 \\
Crawford II & 3 \\
Crawford III & 5 \\
Crawford IV & 1 \\
Crawford V & 11 \\
PAAA & 9 \\
Anastomotic aneurysm & $24.8 \pm 15.1$ \\
Logistic euroscore (\%) [28] & $3.1 \pm 0.3$ \\
ASA [29] & \\
Treatment & 29 \\
Hybrid (VORTEC and endovascular repair) & 5 \\
Aortic graft & \\
\hline
\end{tabular}

Data are numbers or means \pm standard deviations

$T A A A$ thoracoabdominal aortic aneurysm, PAAA pararenal aortic aneurysms, ASA American Society of Anesthesiologists

either aneurysms morphologically unsuitable for endovascular repair (i.e., four patients) or ruptured aneurysm (i.e., one patient) open graft repair was performed simultaneously to the visceral revascularisation (same narcosis). Patient characteristics are displayed in Table 1. Median follow-up was 43 (range 9-89) months. The study had institutional review board approval, and written consent was waived.

\section{Surgical Technique}

A total of 63 renovisceral arteries (54 renal arteries and nine visceral arteries) were revascularized using VORTEC. The technique has already been described by our group $[12,13]$. Briefly, to perform VORTEC, the branch vessel is minimally exposed and punctured, usually about a centimetre distal to its origin; the graft device (Hemobahn/Viabahn, W.L. Gore \& Associates, Flagstaff, AZ) is introduced using the Seldinger Technique. When the stent-graft is partially inside the aortic branch and partially outside, it is expanded by balloon dilatation. The proximal end of the stent is sutured end to side to the main graft or deployed within a vascular graft already connected to the vascular system. The second, more advanced technique allows immediate restoration of blood flow to the branch vessel and thus shorter ischemia time $(<1 \mathrm{~min})$, because the proximal end to side suture to the main graft is no longer required [13]. Finally, in most cases the proximal end of the target artery is ligated at its origin to prevent backflow into the aorta. For renal arteries, 5-7 mm/ $5-\mathrm{cm}$ Viabahn are mostly used, whereas for mesenteric artery or celiac trunk $7-10 \mathrm{~mm} / 5-\mathrm{cm}$ Viabahn more often are employed. 


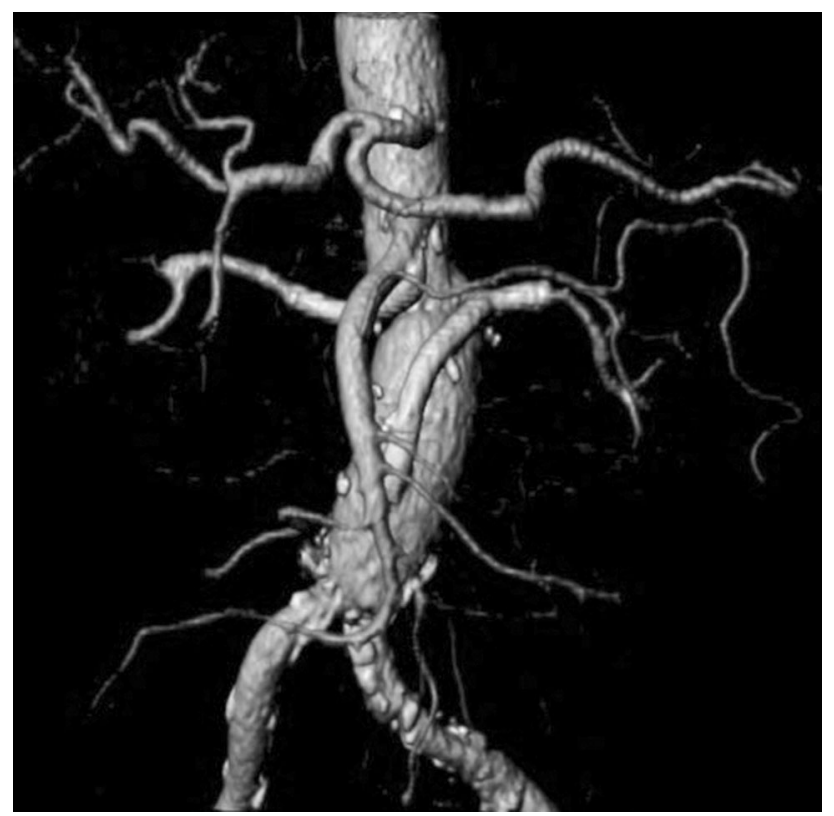

Fig. 1 Volume rendering (VR) of a 77-year-old male patient after revascularization of both renal arteries with Viabahn Open Revascularization Technique (VORTEC) and open graft repair following PAAA. The right renal stent-graft (6-mm Viabahn) has been implanted into the superior mesenteric artery and the left renal stent-graft (6-mm Viabahn) into the aortic graft

Image Technique

CT scans were obtained with two different 64-slice CT scanners (Somatom Definition AS/Sensation 64, Siemens Healthcare, Germany). One hundred milliliters of nonionic iodinated contrast material (Iopromide, Ultravist 300, $300 \mathrm{mg}$ iodine/ml; Bayer Schering Pharma, Germany) was used. Tube voltage was 100 or $120 \mathrm{kV}$, reference tube current ranged from 110 to $210 \mathrm{mAs}$. Images were reconstructed with a slice thickness of $2 \mathrm{~mm}$, and an increment of $1.6 \mathrm{~mm}$ with a medium smooth tissue convolution kernel (B30f). Dedicated software (3mensio, The Netherlands) was used, allowing precise measurement and characterisation of the vessels.

Before intervention, the image protocol only included an arterial phase. Following intervention (in the first week, at 6 weeks, at 3, 6, and 12 months, and then annually), the imaging protocol included an arterial and a venous phase scan, and a native scan for detection of calcifications only done at the first follow-up exam. A postinterventional CTA (within 1 month) and at least one follow-up exam at 6 months or more after intervention (median follow-up 43 months, range 9-89 months) were available in all patients.

Two patients were followed with MRA due to renal insufficiency. MRA were acquired on a 1.5-T MRI system (Signa HD Echo Speed, GE Healthcare, Milwaukee, WI). Imaging protocol included unenhanced and contrastenhanced sequences. A gadoterate meglumine (Dotarem, Geurbet, Roissy, France) dose of $0.1 \mathrm{mmol} / \mathrm{kg}$ of body weight or a gadobutrol (Gadovist, Bayer Schering Pharma, Germany) dose of $0.1 \mathrm{mmol} / \mathrm{kg}$ body weight were used for MRA. A coronal arterial and a venous phase were acquired, each within a single breath hold.

Postprocessing technique included multiplanar reformation (MPR), curved MPR, and volume rendering (VR). MPR is a technique that enables the viewer to generate cross-sections in any plane. A curved plane provides a curved MPR, useful for more accurate assessment of tortuous vessels [15]. VR is commonly used to generate a volumetric display by rendering the entire volume of data and is especially useful for better illustration of complex vascular anatomy (Fig. 1).

Image assessment

Two radiologists retrospectively assessed pre- and postinterventional image examinations in consensus. On the preinterventional CTA, kidney size was assessed. On postinterventional image studies bypass occlusion (defined as complete occlusion), bypass stenosis (endoluminal narrowing) (graded as following; $<50 \%, 50-70 \%$, and $>70 \%$ ), stent-graft dislocations, infolding, stent-graft fractures, and kidney size was assessed. Kidney shrinkage was defined as a change in size of more than $1 \mathrm{~cm}$ and an interpole distance of less than the $10 \%$ percentile of normal value, according to gender and side $(10 \%$ percentile of normal kidney length, men, right side, $99.0 \mathrm{~mm}$; men, left side, $100.0 \mathrm{~mm}$; women, right side, $90.31 \mathrm{~mm}$; women, left side, $92.12 \mathrm{~mm}$ ) [16].

\section{Mortality}

Mortality of patients during follow-up was assessed using the medical report (KISIM, clinic information system, Cistec AG, Switzerland). Thirty-day mortality was not assessed, because we only included patients with at least one follow-up image after 6 months or more.

\section{Statistical Analysis}

Statistical analysis was performed using SPSS (SPSS, release 18.0 for Windows; SPSS, Chicago, IL). Results for continuous variables were reported as mean \pm standard deviation (SD). Cumulative stent-graft patency rate was estimated by using Kaplan-Meier life table analysis.

\section{Results}

\section{Bypass Occlusion}

Cumulative patency rate (i.e., stent-grafts with no occlusion) was $95.2 \pm 2.7 \%$ at 12 months, $87.7 \pm 4.4 \%$ at 24 


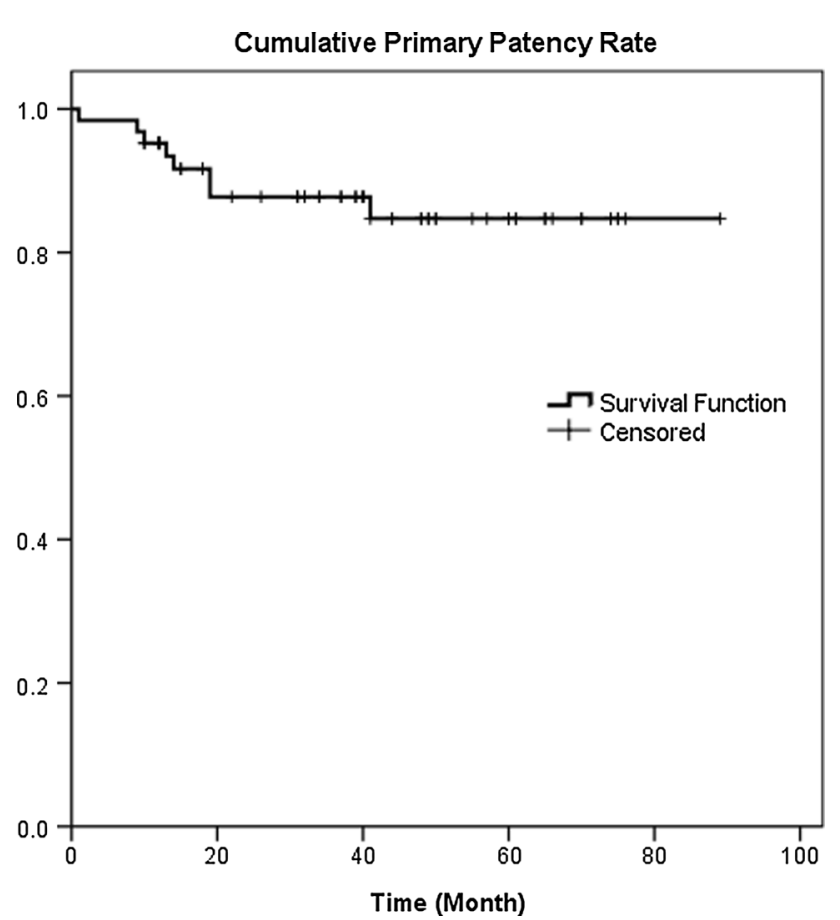

Fig. 2 Graph showing cumulative primary patency rate of stentgrafts over the time of follow-up (upto 89 months). Vessels at risk: baseline, 63; 12 months, 57; 24 months, 44; 36 months, 36; 48 months, 24; 60 months, 15; 72 months, 6; 84 months, 1

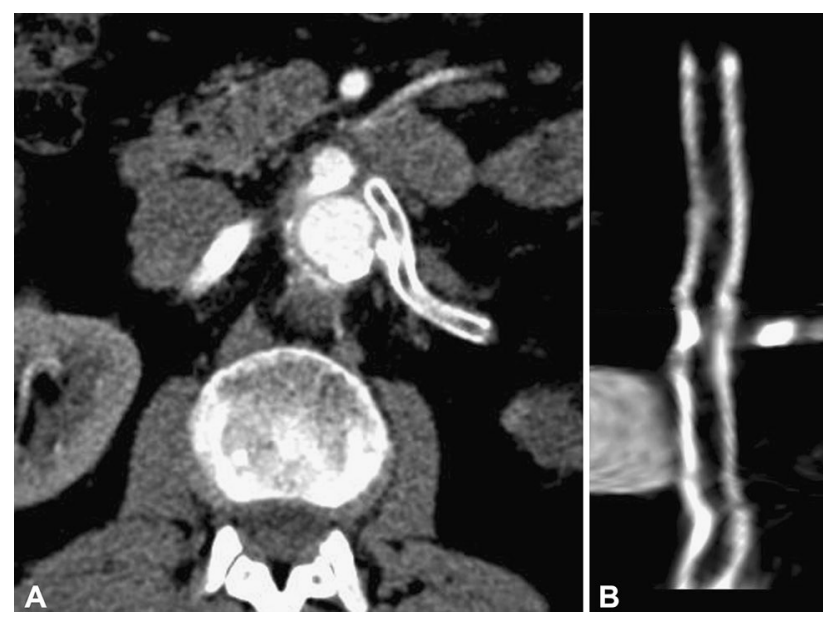

Fig. 3 A Transverse image reconstruction of a 75-year-old male patient shows occlusion of stent-graft to the left renal artery. B Corresponding curved MPR confirming total occlusion

and 36 months, and $84.7 \pm 5.2 \%$ at $48,60,72,84$, and 89 months (Fig. 2). Of the detected stent-graft occlusions (i.e., 8/63), all were to the renal arteries (unilateral, six patients; bilateral, one patient; Fig. 3). Three stent-graft occlusions were without detectable cause, and one patient showed kinking of the stent-graft. These patients did not receive an intervention following stent-graft occlusion. Three patients developed renal stent-graft occlusion in a

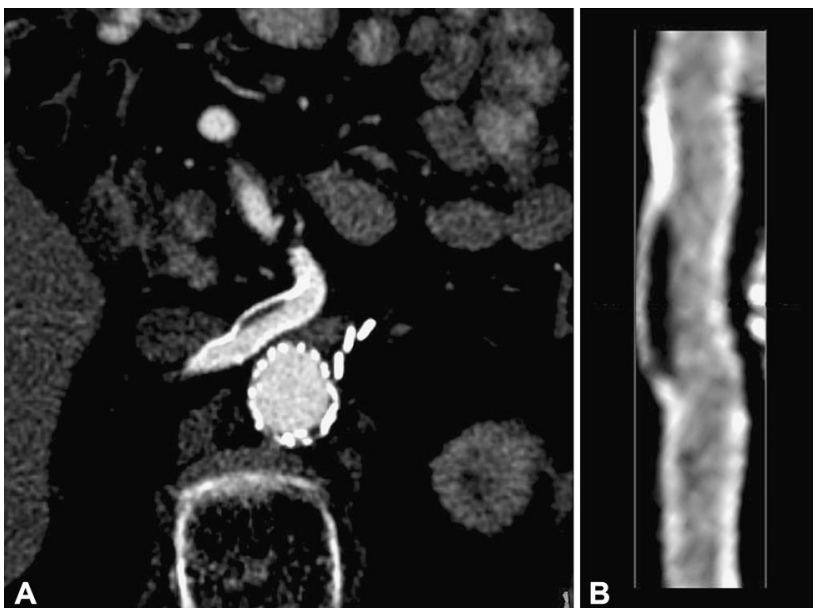

Fig. 4 A Transverse image reconstruction of 83-year old male patient shows eccentric in-stent stenosis $(<50 \%)$ of the stent-graft to the right renal artery. B Corresponding curved MPR

context of probable graft infection associated with aortoenteric fistula. Two of these patients showed unilateral thrombosis (at 9 months; at 41 months), whereas one showed bilateral thrombosis of renal Viabahn (at 19 months), as well as thrombosis of feeding-graft to the superior mesenteric artery (subsequent thrombus aspiration was successful). Nevertheless, the patient died perioperatively due to mesenterial ischemia. The former two patients with graft infection required emergency intervention (partial duodenal resection and repair of aortic graft; thrombin injection of a secondary pseudoaneurysm associated with aortoduodenal fistula), both died following intervention. After 48 months, no additional stent-graft occlusion was observed.

\section{Bypass Stenosis}

Overall, $19 \%$ of stent-grafts (i.e., 12/63) developed bypass stenosis during follow-up $(<50 \%, 10$ stent-grafts; $50-70 \%, 1$ stent-graft; $>70 \%, 1$ stent-graft; Fig. 4). Two patients developed stenosis (both $<50 \%$ ) in stent-grafts to the superior mesenteric artery and to the coeliac trunk within 1 month, which resolved spontaneously. Within 12 months, one visceral stent-graft and one renal stentgraft showed stenosis (visceral stent-graft, $<50 \%$; renal stent-graft, $<50 \%$ ); the renal stenosis resolved spontaneously. These "vanishing stenosis" most likely represented mural thrombi that spontaneously lyse. Within 24 months, three more renal stent-grafts showed stenosis (all $<50 \%$ ), one showed increasing stenosis within 1 year more (50-70\%), and total occlusion at 41 months. Within 36 months, three more renal stent-grafts developed stenosis ( 1 stent-graft, $>70 \% ; 2$ stent-grafts, $<50 \%$ ). In the more severe case, balloon dilatation and stenting were 
successful. The patient died 5 months later of unknown cause. Renal bypass stenosis was noted in another patient within 48 months $(<50 \%)$ and within 96 months $(<50 \%)$, respectively.

Stent-graft Dislocation, Infolding of Stent-graft, and Fracture

No stent-graft used for renovisceral revascularization showed dislocation or infolding. Furthermore, no stentgraft fracture was observed.

\section{Kidney Shrinkage}

Nine patients (i.e., 26.5\%) showed kidney shrinkage (unilateral, 8 patients; bilateral, 1 patient). Seven cases were due to occlusion of renal VORTEC: one after encroachment of a renal branch. One patient showed kidney shrinkage due to occlusion of a renal artery that was not revascularised by VORTEC. Another case was due to occlusion of a renal bypass following redo EVAR and secondary renal bypass after proximal neck aneurysm ( 2 months after open graft repair and revascularisation of renal artery on the other side).

\section{Mortality}

Of the 34 patients included in this study, 11 (i.e., $32.3 \%$ ) died during the follow-up period. Three patients died following graft infection and associated aortoenteric fistula (at 9 months; at 19 months; at 41 months), and two patients died following gastrointestinal bleeding (at 12 months; at 34 months). One patient died due to bowel ischemia, without occlusion of renovisceral stent-grafts (at 15 months). One patient died from pneumonia in the context of bronchial carcinoma (at 26 months) and one from respiratory insufficiency (at 31 months). Three patients died of unknown cause.

\section{Discussion}

Despite the improvement of surgical techniques and strategies, revascularization of renovisceral vessels using conventional suture remains challenging, time-consuming, and prone to complications. Especially in high-risk patients with increasing age and comorbidities, organ ischemia and time-consuming procedures can lead to major postoperative complications $[2,11]$. In this context, a sutureless and clampless anastomosis technique based on the use of the Viabahn has been recently developed to simplify renovisceral debranching and to reduce end-organ ischemia time [13]. First studies have shown promising short- and mid- term results [7, 14, 17]; however, there are no reports about long-term outcome of stent-grafts used for this indication. Our study retrospectively evaluated the long-term outcome of the Viabahn used for VORTEC in a cohort of 34 patients based on CTA and MRA, with a follow-up of up to 89 months.

Recent studies [7, 12-14, 17] showed good short- and mid-term patency rates. In this study, we demonstrated that stent-graft patency rates remained high over a median follow-up of 43 months (at 89 months $84.7 \pm 5.2 \%$ ), which supports the viability of this novel surgical approach. Furthermore, after 48 months no additional stent-graft occlusion was observed. Endoluminal narrowing was seen in $19 \%$ of stent-grafts; only one showed stenosis $>70 \%$. VORTEC has been used mostly in renovisceral vessels considered hostile for standard suturing technique. A majority of targeted vessels showed diffuse disease and were of small diameter. In such vessels, flow profiles might have a high-risk for thrombosis and stenosis. Furthermore, it is worthwhile to mention that in these very first VORTEC cases the older generation Viabahn with no heparin coating was used. Recently, only the Viabahn with a stable and long-time acting Carmeda coating has been used. In addition, optimal platelet inhibition and anticoagulation regimens for both acute and long-term applications have not been defined for renovisceral surgery or in the case where VORTEC is used.

Secondary stent-graft dislocation has been another concern; however, in this study there was no renovisceral stent-graft migration and no stent-graft fracture. Thus, the use of this technique may be extended, and recent studies have shown excellent results employing VORTEC to the supra-aortic vessels $[17,18]$ as well as to the above-knee femoropopliteal anastomosis [19, 20].

Alternative treatment options for aortic aneurysm include fenestrated and chimney endografts or open aneurysm repair. Recent studies have shown 1-year survival rates of 57-89.1\% for patients treated with chimney technique and of 85-93\% for patients treated with fenestrated endografts, and 5-year survival rates of $60 \%$ for patients treated with fenestrated endografts [21-23]. For elective open aneurysm repair, reported survival rates at 1 year are 73-89 \% and at 5-6 years 69.9-84.5 \% [2, 2426]. In our study, we only included patients who had follow-up imaging after 6 months or more; however, in a previous study our group assessed mortality rate in patients electively treated with VORTEC as part of the hybrid procedure, which has shown to be $8.6 \%$ in 30 days and $25.8 \%$ during a mean follow-up of 22.1 months. [7]. Recent studies also have shown excellent results expanding the use of VORTEC, employing VORTEC to the supraaortic vessels [17, 18], as well as to the above-knee femoropopliteal anastomosis [19, 20]. 
The main limitation of our study is that we only assessed Viabahn in patients with at least one imaging follow-up after 6 months or more, and therefore stent-graft complications in patients who did not survive the first 6 months or were lost to follow-up were not taken into account. We refer to another publication that already addressed this topic [7]. Furthermore, this was a retrospective study with only a limited number of high-risk patients, and the patient group was heterogeneous with respect to risk factors and treatment.

Concerning assessment of the kidneys, we only analysed the change in size and not focal infarction or renal function. Thus, change in size due to other factors, such as chronic kidney diseases, cannot be excluded.

\section{Conclusions}

The use of VORTEC in patients with TAAA or PAAA might be an effective way to maintain blood flow to the renovisceral arteries. CTA- and MRA-based results show good long-term patency of stent-grafts from 6 to 89 months and no stent-graft migration or fraction. However, endoluminal narrowing can be observed in almost $20 \%$ of stent-grafts, and close surveillance is required.

Conflict of Interest Anna Winklehner, Thi Dan Linh Nguyen-Kim, Thomas Pfammatter, Zoran Rancic, Dieter Mayer, Mario Lachat and Thomas Frauenfelder have no conflict of interest to declare.

Statement of Informed Consent The study had institutional review board approval. Written consent was waived due to retrospective character of the study.

Statement of Human Rights For this type of study, formal consent is not required.

\section{References}

1. Zarins CK, Harris EJ Jr (1997) Operative repair for aortic aneurysms: the gold standard. J Endovasc Surg 4(3):232-241

2. Patel R, Conrad MF, Paruchuri V, Kwolek CJ, Chung TK, Cambria RP (2009) Thoracoabdominal aneurysm repair: hybrid versus open repair. J Vasc Surg 50(1):15-22

3. Cowan JA Jr, Dimick JB, Henke PK, Huber TS, Stanley JC, Upchurch GR Jr (2003) Surgical treatment of intact thoracoabdominal aortic aneurysms in the United States: hospital and surgeon volume-related outcomes. J Vasc Surg 37(6):1169-1174

4. Conrad MF, Crawford RS, Davison JK, Cambria RP (2007) Thoracoabdominal aneurysm repair: a 20 -year perspective. Ann Thorac Surg 83(2):S856-861; discussion S890-852

5. Kabbani LS, Criado E, Upchurch GR Jr et al (2010) Hybrid repair of aortic aneurysms involving the visceral and renal vessels. Ann Vasc Surg 24(2):219-224

6. Quinones-Baldrich WJ, Panetta TF, Vescera CL, Kashyap VS (1999) Repair of type IV thoracoabdominal aneurysm with a combined endovascular and surgical approach. J Vasc Surg 30(3):555-560

7. Donas KP, Lachat M, Rancic Z et al (2009) Early and midterm outcome of a novel technique to simplify the hybrid procedures in the treatment of thoracoabdominal and pararenal aortic aneurysms. J Vasc Surg 50(6):1280-1284

8. Zhou W, Reardon M, Peden EK, Lin PH, Lumsden AB (2006) Hybrid approach to complex thoracic aortic aneurysms in highrisk patients: surgical challenges and clinical outcomes. J Vasc Surg 44(4):688-693

9. Patel HJ, Upchurch GR Jr, Eliason JL et al (2010) Hybrid debranching with endovascular repair for thoracoabdominal aneurysms: a comparison with open repair. Ann Thorac Surg 89(5): 1475-1481

10. Bakoyiannis C, Kalles V, Economopoulos K, Georgopoulos S, Tsigris C, Papalambros E (2009) Hybrid procedures in the treatment of thoracoabdominal aortic aneurysms: a systematic review. J Endovasc Ther 16(4):443-450

11. Da Rocha MF, Miranda S, Adriani D, Urgnani F, Riambau VA, Mulet J (2009) Hybrid procedures for complex aortic pathology: initial experience at a single center. Rev Esp Cardiol 62(8):896-902

12. Lachat M (2010) Sutureless anastomosis: How to do them. The Vortec Technique. Controversies and Updates in Vascular Surgery; Paris, France

13. Lachat M, Mayer D, Criado FJ et al (2008) New technique to facilitate renal revascularization with use of telescoping selfexpanding stent grafts: VORTEC. Vascular 16(2):69-72

14. Papadimitriou D, Mayer D, Lachat M et al (2012) A clampless and sutureless aortic anastomosis technique using an endograft connector for aortoiliac occlusive disease in which the aorta cannot be clamped or sewn due to calcification or scarring. Vascular 20(5):262-267

15. Krauss T, Pfammatter T, Mayer D et al (2010) Hybrid-repair of thoraco-abdominal or juxtarenal aortic aneurysm: what the radiologist should know. Eur Radiol 20(4):1011-1022

16. Glodny B, Unterholzner V, Taferner B et al (2009) Normal kidney size and its influencing factors-a 64-slice MDCT study of 1.040 asymptomatic patients. BMC Urol 9:19

17. Rancic Z, Mayer D, Pfammatter T et al (2010) A new sutureless telescoping anastomotic technique for major aortic branch revascularization with minimal dissection and ischemia. Ann Surg 252(5):884-889

18. Donas KP, Rancic Z, Lachat M et al (2010) Novel sutureless telescoping anastomosis revascularization technique of supraaortic vessels to simplify combined open endovascular procedures in the treatment of aortic arch pathologies. J Vasc Surg 51(4):836-841

19. Szendro G, Greenberg G, Leytzin A, Mayzler O, Ginzburg V (2011) A new minimally invasive hybrid technique for femoroabove knee popliteal bypass. Int Angiol 30(6):522-526

20. Greenberg G, Szendro G, Mayzler O, Ginzburg V, Leytzin A (2011) Use of ViaBahn open revascularisation technique for above-knee femoro-popliteal anastomosis: a technical note. Eur J Vasc Endovasc Surg 42(2):202-205

21. Suominen V, Pimenoff G, Salenius J (2013) Fenestrated and chimney endografts for juxtarenal aneurysms: early and midterm results. Scand J Surg 102(3):182-188

22. Kristmundsson T, Sonesson B, Dias N, Tornqvist P, Malina M, Resch T (2014) Outcomes of fenestrated endovascular repair of juxtarenal aortic aneurysm. J Vasc Surg 59(1):115-120

23. Bruen KJ, Feezor RJ, Daniels MJ, Beck AW, Lee WA (2011) Endovascular chimney technique versus open repair of juxtarenal and suprarenal aneurysms. J Vasc Surg 53(4):895-904; discussion 904-895 
24. Hinterseher I, Kuffner H, Koch R, Gabel G, Saeger HD, Smelser D (2012) Comparison of survival rates for abdominal aortic aneurysm treatment methods. World J Surg 36(4):917-922

25. Ito H, Shimono T, Shimpo H, Kato N, Takeda K (2013) Open surgery for abdominal aortic aneurysm in the era of endovascular repair: comparison with long term results of endovascular repair using zenith stentgraft. Ann Vasc Dis 6(2):189-194

26. De Bruin JL, Baas AF, Buth J et al (2010) Long-term outcome of open or endovascular repair of abdominal aortic aneurysm. N Engl J Med 362(20):1881-1889
27. Safi HJ, Miller CC 3rd (1999) Spinal cord protection in descending thoracic and thoracoabdominal aortic repair. Ann Thorac Surg 67(6):1937-1939; discussion 1953-1938

28. Roques F, Michel P, Goldstone AR, Nashef SA (2003) The logistic EuroSCORE. Eur Heart J 24(9):881-882

29. American Society of Anesthesiologists (1963) New classification of physical status. Anesthesiology 24:111 